\section{Clostridium difficile Colonization of Nursing Home Residents}

To the Editor-Clostridum difficile is a leading cause of infectious diarrhea in nursing homes. ${ }^{1}$ Evidence-based infection control guidelines are needed to reduce transmission of C. difficile in nursing homes. These guidelines should account for the prevalence of $C$. difficile in the nursing home environment. The primary objective of this project was to assess the proportion of nursing home residents colonized with toxigenic C. difficile.

A random sample of community-based nursing home residents $(\mathrm{n}=40 ; 10 \%)$ and Veterans Affairs Community Living Center (VA CLC) residents $(\mathrm{n}=40 ; 20 \%)$ were selected retrospectively from 2 cohort studies on MRSA colonization and transmission. ${ }^{2,3}$ The studies enrolled 401 residents from 13 community-based nursing homes in Maryland and Michigan and 200 residents from 5 VA CLCs in 4 states and the District of Columbia. Selection within the community-based facilities was designed to be representative of all nursing home residents. However, in the VA CLCs, 2 groups of residents were enrolled: residents with a recent (within 1 year) history of MRSA colonization and residents without recent MRSA colonization. All VA CLC residents with recent MRSA colonization were approached for enrollment. A random sample of residents without recent MRSA colonization was approached for enrollment to provide a representative sample. Specimens from the perianal skin were taken from enrolled residents. Notably, diarrhea was reported for $2 \%-3 \%$ of the study participants. No C. difficile outbreaks were reported during the studies.

Culture-based methods were used to detect toxigenic C. difficile in perianal swabs. Resident swabs from the perianal skin were placed in cycloserine cefoxitin mannitol broth with taurocholate and lysozyme broth (Anaerobe Systems; Morgan Hill, CA) at $35^{\circ} \mathrm{C}$ in anaerobic conditions, and growth was observed at 24 hours, 48 hours, and 7 days. If growth was observed, the culture was transferred to a blood agar plate and incubated in aerobic conditions at $35^{\circ} \mathrm{C}$ for 48 hours. Any bacteria growth was identified using RapID Ana II system (Remel, Lenexa, KS). Toxins A and B and C. difficile glutamate dehydrogenase detection were determined using C Diff Quik Chek Complete kits (TechLab, Blacksburg, VA). ${ }^{4}$

Among the community-based nursing homes residents, 1 of 40 residents had perianal skin swabs that tested positive for toxigenic C. difficile (2.5\%; 95\% CI, $0.1 \%-13.2 \%)$. None of the 40 VA CLC residents tested positive for toxigenic $C$. difficile (0\%; 95\% CI, 0.0\%-8.8\%).

These rates are slightly lower than those reported in the literature. Based on data from 9 eligible studies that included 1,371 subjects, a recent systematic review found that $14.8 \%$ (95\% CI, $7.6 \%-24.0 \%)$ of LTCF residents are asymptomatic carriers of toxigenic $C$. difficile. ${ }^{5}$ The systematic review included
21 LTCFs across 4 countries and 4 states. In contrast, our populations covered 18 nursing homes in 6 states. The facilities in the review with the highest reported rates of $C$. difficile colonization had also experienced recent outbreaks of C. difficile infection, which increased their estimates. Our results should reassure nursing homes that prevalence of toxigenic C. difficile is low during endemic periods. Standard precautions should be sufficient to prevent transmission under nonepidemic conditions.

\section{ACKNOWLEDGMENTS}

Financial support. This work was funded through the University of Maryland's CDC EpiCenter (grant no. 1U54CK000450), an Agency for Healthcare Quality and Research (AHRQ) award (grant no. 1R18HS019979-01A1), and a Merit Review Award (grant no. \#IIR10-154) from the US Department of Veterans Affairs Health Services Research and Development Service. Student support for E.M.S. was provided by an NIGMS Initiative for Maximizing Student Development grant (grant no. 2 R25-GM55036).

Potential conflicts of interest. All authors report no conflicts of interest relevant to this article.

Disclaimer. The contents do not represent the views of the US Department of Veterans Affairs or the US Government. Clinical Trial Registration No.: NCT01350479.

\section{Mary-Claire Roghmann, MD, MS; $;$ Liana R. Andronescu, MPH; ${ }^{1}$ Emily M. Stucke, BA; ${ }^{1}$ J. Kristie Johnson, $\mathrm{PhD}^{3}$}

Affiliations: 1. Department of Epidemiology and Public Health, University of Maryland School of Medicine, Baltimore, Maryland; 2. Geriatrics Research Education and Clinical Center, Veterans Affairs Maryland Health Care System, Baltimore, Maryland; 3. Department of Pathology, University of Maryland School of Medicine, Baltimore, Maryland.

Address correspondence to Mary-Claire Roghmann, MD, MS, University of Maryland School of Medicine, 10 South Pine Street, MTSF Room 336, Baltimore, MD 21201 (mroghman@epi.umaryland.edu).

Infect Control Hosp Epidemiol 2017;38:1267-1268

(c) 2017 by The Society for Healthcare Epidemiology of America. All rights reserved. 0899-823X/2017/3810-0025. DOI: $10.1017 /$ ice.2017.172

\section{REFERENCES}

1. Jump RLP, Donskey CJ. Clostridium difficile in the long-term care facility: prevention and management. Curr Geriatr Rep 2015;4:60-69.

2. Roghmann MC, Johnson JK, Sorkin JD, et al. Transmission of methicillin-resistant Staphylococcus aureus (MRSA) to healthcare worker gowns and gloves during care of nursing home residents. Infect Control Hosp Epidemiol 2015;36:1050-1057.

3. Pineles L, Morgan DJ, Lydecker A, et al. Transmission of methicillin-resistant Staphylococcus aureus to health care worker gowns and gloves during care of residents in Veterans Affairs nursing homes. Am J Infect Control 2017. doi: 10.1016/ j.ajic.2017.03.004.

4. Hink T, Burnham CD, Dubberke ER. A systematic evaluation of methods to optimize culture-based recovery of Clostridium difficile from stool specimens. Anaerobe 2013;19:39-43. 
5. Ziakas PD, Zacharioudakis IM, Zervou FN, Grigoras C, Pliakos EE, Mylonakis E. Asymptomatic carriers of toxigenic C. difficile in longterm care facilities: a meta-analysis of prevalence and risk factors. PLoS One 2015;10:e0117195.

\section{Comparison of Rates of Drain-Related Ventriculitis According to Definitions Used}

To the Editor-We read with interest the recent comparison of ventricular drain-related ventriculitis (VDRV) according to which definitions were used. ${ }^{1}$ Reyes et $\mathrm{al}^{1}$ undertook a retrospective assessment of 52 cases of VDRV using 4 sets of definitions and found that using the National Healthcare Safety Network (NHSN) definitions resulted in substantially more cases of DDRV being identified.

We recently undertook a pilot study as a prelude to the introduction of a national surveillance system of VDRV, and as part of that process, we reviewed many definitions. ${ }^{2}$ However, because overreliance on culture can occur, we categorized VDRV as either definite or probable and allowed for the fact that bacteria isolated from the cerebrospinal fluid (CSF) could represent contamination (eg, a single sample with coagulase negative staphylococci). ${ }^{2}$ Of 45 cases of VDRV in 4 pilot centers, 20 of 28 cases categorized were definite.

Meningitis or ventriculitis following neurosurgery is complex, and the infection rate can be expressed as a percentage of patients with a drain inserted or preferably as the rate of infection per 1,000 catheter days. ${ }^{3-5}$ The latter metric better reflects the risk associated with device duration. Ramanan et $\mathrm{al}^{5}$ reviewed 35 studies, which included 752 infections, and found that the rate was lower for high-quality studies than for lower-quality studies. This effect contrasts with that found with most other infections, where better surveillance identifies higher rates of infection. This finding highlights the complexity of this area of study.

In a literature search of definitions used to diagnose VDRV, 16 unique definitions were retrieved. ${ }^{6}$ A positive CSF culture was required in $50 \%$ of these definitions, but no definitions mandated that more than 1 CSF culture be positive to confirm infection. However, only 7 of 16 definitions (44\%) were objective, that is, they relied on laboratory data and clinical findings that were not overly open to interpretation. ${ }^{6}$ This finding explains, in large part, the variation in infection rates described in the literature.

The decision to start antibiotics in a neurosurgical patient with a drain in situ is largely a clinical one and must be guided by the best interest of the individual patient. This often means that more patients with suspected ventriculitis or meningitis are empirically treated than are subsequently confirmed to have the infection. In a recent study from the Netherlands, 48 of 209 patients with suspected ventriculitis
(23\%) were started on empirical antibiotics after subarachnoid haemorrhage. ${ }^{7}$ However, in only 11 patients $(5 \%)$ were the CSF cultures positive. A high red blood cell count in the CSF, as might perhaps be expected in this group of patients, was statistically associated with CSF culture-negative cases. $^{7}$

While the greater availability of molecular methods to diagnose VDRV may assist in determining the microbial cause, there will always be a need to assess a combination of clinical features, microbiological results, and other CSF parameters such as a protein levels, glucose levels, and cell counts. Surveillance definitions, however, should allow for the complex nature of this condition and the difficulties in being certain of the diagnosis. Not all cases are clear cut, and a positive CSF culture does not always indicate VDRV, especially if the case involves an organism that may reside on the skin, hence, the higher rate of VDRV with NHSN definitions. ${ }^{1}$ Consequently, there is a need for international agreement on surveillance definitions that are practicable and as rigorous as possible. Establishing such definitions will facilitate comparisons between centers that can inform improvements in the care related to these invasive devices and, ultimately, in patient outcomes.

\section{ACKNOWLEDGMENTS}

Financial support: No financial support was provided relevant to this article.

Potential conflicts of interest: All authors report no conflicts of interest relevant to this article.

\section{Hilary Humphreys, MD, FRCPI, FRCPath; ${ }^{1,2}$ Peter J. Jenks, MD, MRCP, FRCPath; ${ }^{3}$ Jennie Wilson, $\mathrm{PhD}^{4}$}

\begin{abstract}
Affiliations: 1. Department of Clinical Microbiology, The Royal College of Surgeons in Ireland, Dublin, Ireland; 2. Department of Microbiology, Beaumont Hospital, Dublin, Ireland; 3. Department of Microbiology and Infection Prevention and Control, Derriford Hospital, Plymouth, United Kingdom; 4. Richard Wells Research Centre, University of West London, London, United Kingdom.

Address correspondence to Hilary Humphreys, MD, FRCPI, FRCPath, Department of Clinical Microbiology, RCSI Education and Research Centre, Beaumont Hospital, Dublin D09 YD60 (hhumphreys@rcsi.ie). Infect Control Hosp Epidemiol 2017;38:1268-1269

(C) 2017 by The Society for Healthcare Epidemiology of America. All rights reserved. 0899-823X/2017/3810-0026. DOI: 10.1017/ice.2017.175
\end{abstract}

\section{REFERENCES}

1. Reyes MM, Munigala S, Church EL, et al. Comparing external ventricular drains-related ventriculitis surveillance definitions. Infect Control Hosp Epidemiol 2017;38:574-579.

2. Humphreys H, Jenks P, Wilson J, et al. Surveillance of infection associated with external ventricular drains; proposed methodology and results from a pilot study. J Hosp Infect 2017;95:154-160.

3. van de Beek D, Drake JM, D, Tunkel AR. Nosocomial bacterial meningitis. N Eng J Med 2010;362:146-154. 